Synthesis of Natural

Products and

Potential Drugs

\title{
Asymmetric Fluorination Approach to an SYK Inhibitor
}
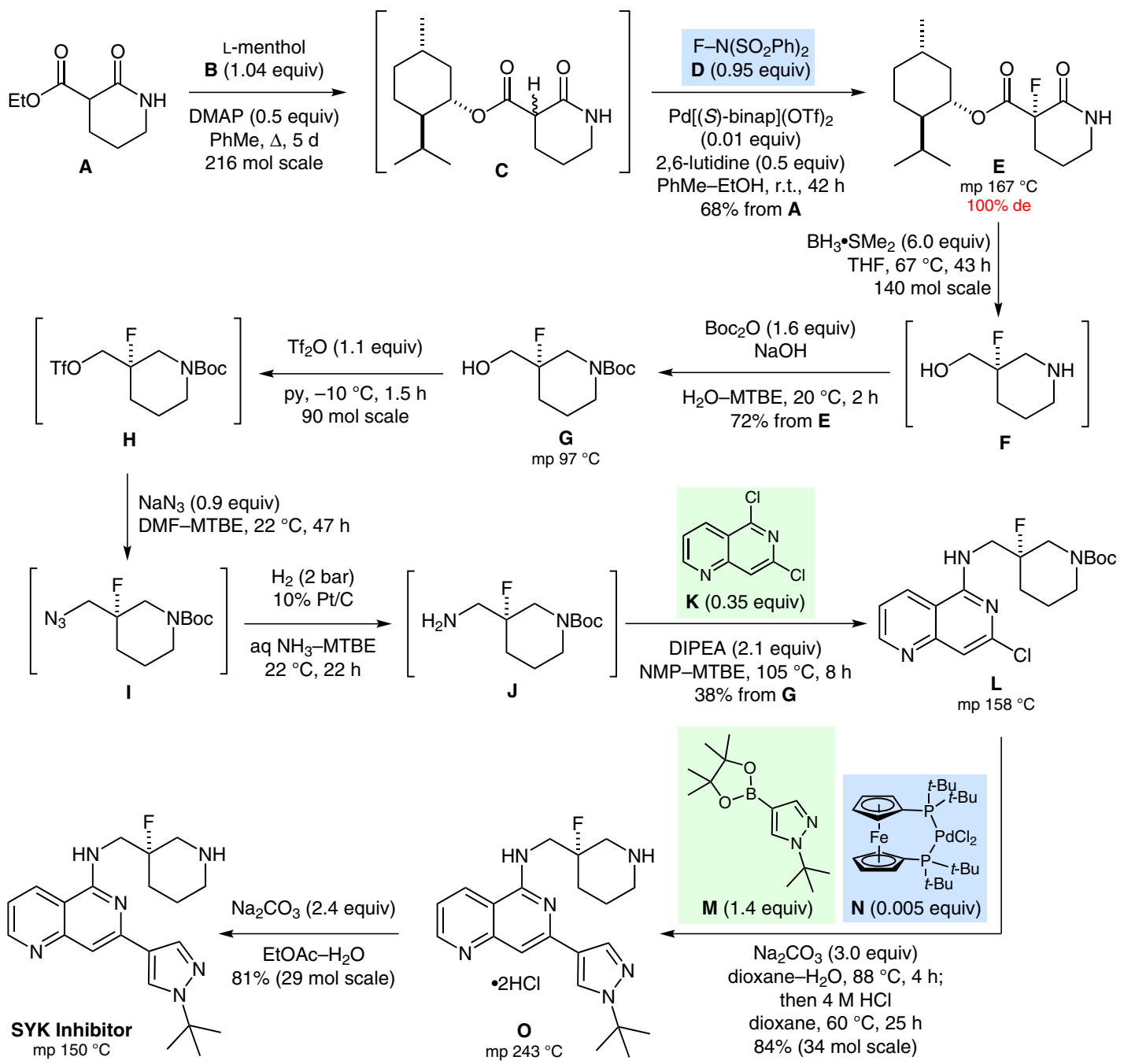

\section{Key words}

\section{SYK inhibitor}

asymmetric

fluorination

N-fluorobenzene-

sulfonimide

palladium catalysis

Suzuki-Miyaura cross-coupling

\section{Synfacth of the}

Significance: Spleen tyrosine kinase (SYK) is implicated in diverse cellular responses such as proliferation, differentiation, and phagocytosis. The target molecule is a SYK inhibitor that is of interest for the treatment of rheumatoid arthritis, B-cell lymphoma, and asthma. The highly telescoped, large-scale synthesis depicted delivered eight kilograms of API.
Comment: The asymmetric fluorination of $\beta$-keto ester $\mathbf{A}$ using (S)-BINAP as the chiral ligand gave a modest $44 \%$ ee but this improved to $72 \%$ ee with the bulkier DTBM-SEGPHOS ligand. The best results were obtained by the combined use of a chiral auxiliary (L-menthol) and an enantio- and diastereoselective fluorination $(\mathbf{C}+\mathbf{D} \rightarrow \mathbf{E})$ mediated by $\operatorname{Pd}[(S) \text {-binap](OTf })_{2}$.

SYNFACTS Contributors: Philip Kocienski

Synfacts 2015, 11(10), 1011 Published online: 18.09.2015

Dol: 10.1055/s-0035-1560258; Reg-No.: K05315SF 